\title{
ANALYSIS OF RISK FACTORS IN PATIENTS WHO HAVE UNDERGONE CORONARY ARTERY BYPASS GRAFTING SURGERY AND VALVE REPLACEMENT SURGERY
}

\author{
Fatih Erkan Akay ${ }^{1}$, Begüm Soyleyici ${ }^{1}$, Alperen Elibol ${ }^{2}$, Pelinsu Elif Hünkar ${ }^{1}$, Berfin Tan ${ }^{1}$, Volkan Yüksel ${ }^{3}$ \\ ${ }^{1}$ Trakya University School of Medicine, Edirne, TURKEY \\ ${ }^{2}$ Acıbadem Mehmet Ali Aydınlar University School of Medicine, Istanbul, TURKEY \\ ${ }^{3}$ Department of Cardiovascular Surgery, Trakya University School of Medicine, Edirne, TURKEY
}

\begin{abstract}
Aims: The aim of the study is to investigate differences in usual cardiovascular risk factors' and comorbidities between the patients who had undergone coronary artery bypass grafting surgery and valve replacement surgery at Cardiovascular Surgery Department of Trakya University School of Medicine. Methods: In this study, patients who had coronary artery bypass grafting surgery and valve replacement surgery at Cardiovascular Surgery Department of Trakya University School of Medicine between 01.01.2018 and 31.12.2018 were analyzed retrospectively. All data regarding the type of cardiovascular operation, habits of smoking, alcohol consumption, family history, chronic diseases and laboratory findings were analyzed using R version 3.5.0. Results: The total number of patients in the study was 130 . There were 106 patients who had coronary bypass grafting surgery, 90 (95.4\%) of them were male and $16(4.6 \%)$ of them were female. The number of the patients who had valve replacement surgery was 24 where $15(62.5 \%)$ of them were female and $9(37.5 \%)$ were male. Differences in patients' high-density lipoprotein levels, fasting blood glucose levels along with gender, habits of smoking, diabetes mellitus status and alcohol consumption between coronary artery bypass grafting surgery and valve replacement surgery groups were found statistically significant. Conclusion: Risk factors for cardiovascular disease are very dynamic and multifactorial. In our analysis, there was a significant difference between risk factors for coronary artery bypass and valve replacement surgeries including high-density lipoprotein levels, fasting blood glucose levels, diabetes mellitus, gender and smoking status. The variability may alter according to the living standards, culture, educational status among patients. Keywords: Coronary disease, coronary artery, surgery, risk factors
\end{abstract}

\section{INTRODUCTION}

Cardiovascular diseases (CVD) are considerable public health issues worldwide. It is estimated that 17.5 million people die from CVD each year (1). Coronary heart disease (CHD) is the building block of CVD and the frequent cause of death in developed countries. Approximately 1.8 million people die from CHD in Europe each year $(1,2)$. Risk factors for ischemic heart disease (IHD) are composed of acute coronary disease, and ischemic stroke according to Global Burden of Disease Study (3). Data from WHO Global Health Observatory were also used to define the prevalence of different cardiometabolic risk factors (3). High levels of body mass index (BMI), glucose, blood pressure, and cholesterol have different effects of death on income group over the time; high-income countries were able to degrade the effects of these risk factors during the past 20 years, whereas lower/middle-income countries have a higher data of an increase in mortality related to high BMI and glucose (4).

Valve diseases are the extensive clinical manifestations of the heart. Yet until this day, their mechanis$\mathrm{ms}$ are inadequately understood. Diagnosis commonly requires the advancement in disease and virtually no acceptable medical options are available in the majority of the cases (5). The active cause is age-related valve degeneration and related developments leading to aortic stenosis and mitral regurgitation $(6,7)$.

The atherosclerotic coronary disease has a multifactorial etiological background with non-modifiable risk factors like age, being a male and genetic predispositi- 
on, numerous other risk factors are changeable depending on individuals lifestyle and/or pharmacotherapy. More than 200 risk factors were defined in Hopkins et al. (8) study in 1981, of which the most relevant ones are held to be high plasma cholesterol level, diabetes mellitus, hypertension, smoking and overweight/ obesity. These risk factors were originally evaluated decades ago based on the data attained from the Framingham study by Dawber et al. (9).

The aim of the study is to investigate differences in usual cardiovascular risk factors' and comorbidities between the patients who had undergone coronary artery bypass crafting surgery (CABGS) and valve replacement surgery (VRS) at Cardiovascular Surgery Department of Trakya University School of Medicine.

\section{MATERIAL AND METHODS}

This retrospective study was approved by the Scientific Research Ethics Committee of Trakya University School of Medicine (TUTF-BAEK2019/224). Informed consent was obtained from all participants. In this study, the data of all patients over 18 years old who had a cardiovascular operation in Trakya University School of Medicine between 1st of January 2018- 31st of December 2018 were analyzed retrospectively. Patients who had multiple surgeries (both CABGS and VRS), patients with missing data and patients operated with procedures other than CABGS and VRS (atrial septal defect repair, pericardiectomy, etc.) were excluded from the study.

Patients' age, gender, habit of smoking, alcohol usage, family history of cardiovascular disease, chronic diseases - if any [hypertension (HTN), diabetes mellitus (DM), chronic obstructive pulmonary disease (COPD), goiter, chronic kidney disease (CKD)], laboratory findings [fasting blood glucose (FBG), low-density lipoprotein (LDL), high-density lipoprotein (HDL), total cholesterol, triglyceride (TG), urea, c-reactive protein (CRP)] and the history of peripheral arterial disease were recorded from the archives of Trakya University School of Medicine.

Data was analyzed using $\mathrm{R}$ version 3.5.0. A p-value $<0.05$ was set for the statistical significance. Continuous variables (age, HDL levels, LDL levels, total cholesterol, FBG, TG levels, CRP and urea levels) were tested for normal distribution with Shapiro-Wilk Test. Non-normal distribution was observed for all continuous variables thus descriptive statistics for those are presented as median and inter-quartile range (IQR). Categorical data (gender, smoking status, alcohol consumption, family history of cardiovascular disease, DM, goiter, COPD,
PAD, CRF, HTN) are presented as numbers and percentages. Mann-Whitney U Test was conducted on continuous and Pearson Chi-Squared Test $\left(\mathrm{x}^{2}\right)$ was conducted on categorical variables.

\section{RESULTS}

In this retrospective study 130 patients ( 31 female, 99 male) who were operated either with CABGS or VRS at Trakya University Department of Cardiovascular Surgery were included.

Median ages of participants were calculated as 63 $(\mathrm{IQR}=13)$, and $64.5(\mathrm{IQR}=15)$ for CABGS and VRS groups, respectively. Differences in median values were not statistically significant $(p=0.787)$. Median HDL levels of participants were calculated as $36(\mathrm{IQR}=9)$ and 46.5 ( $\mathrm{IQR}=13.125$ ) for GABGS and VRS groups, respectively, and this difference was found to be statistically significant $(\mathrm{p}<0.001)$. Median FBG levels of participants was calculated as $111.5(\mathrm{IQR}=51.25)$ and $98.5(\mathrm{IQR}=$ 18.25) for GABGS and VRS groups, respectively. This difference was found to be statistically significant ( $\mathrm{p}=$ 0.007). Median levels of LDL, total cholesterol, triglyceride, CRP and urea between CABGS and VRS groups were not statistically significant $(\mathrm{p}=0.365, \mathrm{p}=0.082$, $\mathrm{p}$ $=0.470, \mathrm{p}=0.535, \mathrm{p}=0.517$, respectively). Summary statistics of continuous variables and Mann-Whitney $U$ Test results presented in Table 1.

Statistically significant interactions between surgery type and gender, smoking status, DM status and alcohol consumption were found $\left(\mathrm{x}^{2}=21.677, \mathrm{p}<0.001 ; \mathrm{x}^{2}\right.$ $=5.386, \mathrm{p}=0.020 ; \mathrm{x}^{2}=4.534, \mathrm{p}=0.033 ; \mathrm{x}^{2}=4.548, \mathrm{p}=$ 0.033; respectively). Males (90.909\%) were more likely to have CABGS than females (51.613\%), and females $(48.387 \%)$ were more likely to have VRS than males (9.091\%). Smokers (90.476\%) were more likely to have CABGS than non-smokers (74.667\%). Patients with DM (90.909\%) were more likely to have CABGS than patients without DM (74.667\%). Patients who consume alcohol (93.023\%) were more likely to have CABGS than patients who do not consume alcohol (80.342\%). $\%)$. Association between surgery type and family history of cardiac diseases, goiter, COPD, CRF, PAD, and HTN was not statistically significant $\left(\mathrm{x}^{2}=0.460, \mathrm{p}=\right.$ $0.498 ; \mathrm{x}^{2}=0.666, \mathrm{p}=0.414 ; \mathrm{x} 2=0.994, \mathrm{p}=0.319 ; \mathrm{x}^{2}=$ $1.500, \mathrm{p}=0.221 ; \mathrm{x}^{2} \sim 0, \mathrm{p} \sim 1 ; \mathrm{x} 2=0.002, \mathrm{p}=0.969$; respectively). Summary statistics of categorical variables and Pearson Chi-Squared Test results are presented in Table 2. 
Table 1: Summary statistics of continuous variables and Mann-Whitney-U Test results.

\begin{tabular}{|c|c|c|c|}
\hline & $\begin{array}{c}\text { CABGS [median } \\
(I Q R)]\end{array}$ & $\begin{array}{l}\text { VRS [median } \\
\quad(I Q R)]\end{array}$ & p value \\
\hline Age (years) & $63(13)$ & $64.5(15)$ & 0.787 \\
\hline$H D L(m g / d L)$ & $36(9)$ & $46.5(13.125)$ & $<0.001$ \\
\hline$L D L(m g / d L)$ & $108(51.75)$ & $109(43.075)$ & 0.365 \\
\hline Total Cholesterol (mg/dL) & $169(64.5)$ & $191.5(58.5)$ & 0.082 \\
\hline$F B G(m g / d L)$ & $111.5(51.25)$ & $98.5(18.25)$ & 0.007 \\
\hline Triglyceride (mg/dL) & $136(59.5)$ & $133(53.5)$ & 0.470 \\
\hline$C R P(m g / d L)$ & $1.17(4.093)$ & $1.29(2.342)$ & 0.535 \\
\hline Urea $(m g / d L)$ & $33(14)$ & $36.5(44.75)$ & 0.517 \\
\hline
\end{tabular}

CABGS: Coronary Artery Bypass Surgery, VRS: Valve Replacement Surgery, IQR: Inter-quartile Range, HDL: High-Density Lipoprotein, LDL: Low-Density Lipoprotein, FBG: Fasting Blood Glucose, CRP: C-reactive protein

Table 2: Summary statistics of categorical variables and Pearson Chi-Squared Test results.

\begin{tabular}{|c|c|c|c|c|c|}
\hline & & $\begin{array}{l}\text { CABGS } \\
{[n(\%)]}\end{array}$ & VRS [n (\%)] & $x^{2}$ & p value \\
\hline \multirow[t]{2}{*}{ Gender } & Female & $16(51.613)$ & $15(48.387)$ & 21.677 & $<0.001$ \\
\hline & Male & $90(90.909)$ & $9(9.091)$ & & \\
\hline \multirow[t]{2}{*}{ Smoking Status } & Smoker & $57(90.476)$ & $6(9.524)$ & 5.386 & 0.020 \\
\hline & Non-smoker & $49(73.134)$ & $18(26.866)$ & & \\
\hline \multirow[t]{2}{*}{$D M$} & Present & 50 (90.909) & $5(9.091)$ & 4.534 & 0.033 \\
\hline & Absent & $56(74.667)$ & $19(25.333)$ & & \\
\hline \multirow[t]{2}{*}{ Family History } & Present & $12(92.308)$ & $1(7.692)$ & 0.460 & 0.498 \\
\hline & Absent & $94(80.342)$ & $23(19.658)$ & & \\
\hline \multirow[t]{2}{*}{ Alcohol Consumption } & Yes & $40(93.023)$ & $3(6.977)$ & 4.548 & 0.033 \\
\hline & No & $66(75.862)$ & $21(24.138)$ & & \\
\hline \multirow[t]{2}{*}{ Goiter } & Present & $0(0)$ & $1(100)$ & 0.666 & 0.414 \\
\hline & Absent & $\begin{array}{c}106 \\
(82.171)\end{array}$ & $23(17.829)$ & & \\
\hline \multirow[t]{2}{*}{ COPD } & Present & $2(50)$ & $2(50)$ & 0.994 & 0.319 \\
\hline & Absent & $104(82.54)$ & $22(17.46)$ & & \\
\hline \multirow[t]{2}{*}{$C K D$} & Present & $10(66.667)$ & $5(33.333)$ & 1.500 & 0.221 \\
\hline & Absent & $96(83.478)$ & $19(16.522)$ & & \\
\hline \multirow[t]{2}{*}{$P A D$} & Present & $2(100)$ & $0(0)$ & $\sim 0$ & $\sim 1$ \\
\hline & Absent & $104(81.25)$ & $24(18.75)$ & & \\
\hline \multirow[t]{2}{*}{$H T N$} & Present & $65(82.278)$ & $14(17.722)$ & 0.002 & 0.969 \\
\hline & Absent & $41(80.392)$ & $10(19.608)$ & & \\
\hline
\end{tabular}

$\boldsymbol{x}^{2}$ : Chi-Squared Test, DM: Diabetes Mellitus, COPD: Chronic Obstructive Pulmonary Disease, PAD: Peripheric Artery Disease, CKD: Chronic Kidney Disease, HTN: Hypertension 


\section{DISCUSSION}

Our study analyzed the prevalence of cardiovascular risk factors among Turkish adults, who had an operation in 2018 in the Cardiovascular Surgery Department of Trakya University School of Medicine, on behalf of investigating morbidity and mortality in coronary artery bypass grafting and valve replacement surgeries.

Gender distribution for CABGS and VRS was statistically significant with a $95.4 \%$ male population in CABGS group that correlates with the risk factors for being a male in CVD (10). Vascular physiological factors such as a narrower atheroma wall, a smaller vessel diameter, less collateral flow, less coronary flow reserve, more vascular rigidity, distinctions in remodeling, and functional variations of smooth muscle cells in the vessel wall of women may be the cause of the difference of the outcomes between men and women (10). Within all age categories, aortic valve sclerosis, and stenosis were more common in males compared with women $(11,12)$. On the other hand, in our study, it was statistically significant for females to have VRS more than the male population.

While low HDL level in CABGS group compared to VRS was significant; LDL, triglyceride and total cholesterol levels were not different between both surgery groups. It is confirmed that the risk of getting CVD is correlated with dyslipidemia, not with total cholesterol levels (13). Dyslipidemia can be described as having unusual values of total cholesterol/LDL, HDL and triglycerides which was present in our study with significance in low HDL levels (13). However, in most studies, in fact, high triglyceride levels is a valuable reason for dyslipidemia (14). The ways of getting a healthy lipid chart may vary; a diet which is rich for vegetables and fruits decrease the risk whereas an eating behavior consists of saturated oils would increase the risk of hyperlipidemia. Daily exercise is another well-known factor for decreasing the chance of hyperlipidemia. Physical exercise improves physical fitness, metabolic profile, and, integrated with sufficient diet significantly reduces body mass and has a beneficial influence on psycho-social status as well as the quality of life (15).

At the same time, having a higher level of FBG and the presence of DM was found to be statistically significant in CABGS group compared to VRS group ( $\mathrm{p}=0.007$ ). The increased FBG level has an effect of accelerating the deposition rate of lipids on the blood vessels, leading to build up of atherosclerotic plaque which has the tendency of building up a thrombus resulting with CHD (16). According to Aronson et al. (17), CHD is an influential determinant of the long-term prediction among patients with DM, associated with a 2 to 4 -fold increased fatality risk from CVD.
In our study, being a smoker and consuming alcohol was statistically significant with having a CVD and undergoing CABGS. Meta-analysis done by Roerecke et al (18) noted that, only people with alcohol use disorder had a high risk of IHD (1.5- to 2-fold). Results also demonstrated that drinkers without any episodic heavy drinking and with an average consumption of $<30 \mathrm{~g} /$ day had the lowest risk of IHD.

Although, high CRP level is found to be a risk factor for CVD, in our study even though both groups had an increased level of CRP, the increase between both groups was not statistically different. In comparison with research run by C-Reactive Protein Coronary Heart Disease Genetics Collaboration (CCGC), increased levels of CRP due to a genetic polymorphism in the CRP encoding DNA segment can be a risk factor for CVD which is compatible with our findings (19).

In order to strengthen the gathered data, number of patients could be increased as well as data could be analyzed and compared with other cardiovascular surgeries. In this study passive smokers were counted to be in the category of non-smokers which may have an effect on our findings. In order to eliminate this effect, a group for passive-smokers might have been created in data collection. In result of earlier research by Law et al (20), it is defined that the addition of confounding factors can be associated with passive smoking and CVD.

Furthermore, advancements in medical science have displayed the pathogenesis of cardiovascular disease to be complex, with a large-scale of underlying factors. Amongst these factors, stress is considered to be crucial (21). Two meta-analyses have done by Rugulies $\mathrm{R}$ (22) and Wulsin et al. (23) indicates that depression has a significantly negative influence on the prognosis of patients with CHD. Therefore patients stress levels and diagnosis with clinical depression could be included.

In conclusion, there was a significant difference between risk factors for CABGS and VRS including HDL levels, fasting blood glucose levels, diabetes mellitus, gender and smoking status. In order to elucidate and amplify the risk factors more being in first place for CABGS and VRS and other cardiovascular surgeries, further studies are in need.

Ethics Committee Approval: This study was approved by the Scientific Research Ethics Committee of Trakya University

School of Medicine (TUTF-BAEK2019/224)

Informed Consent: Written informed consent was obtained from the participants of this study.

Conflict of Interest: The authors declared no conflict of interest.

Author contributions: Concept: FEA, BS, AE, PEH, BT, VY. Design: FEA, BS, AE, PEH, BT, VY. Supervision: FEA, BS, AE, PEH, BT, VY. 
Resources: FEA, BS, AE, PEH, BT, VY. Materials: FEA, BS, AE, PEH, BT, VY. Data collection and/or processing: FEA, BS, AE, PEH, BT, VY. Analysis and/or interpretation: FEA, BS, AE, PEH, BT, VY. Literature search: FEA, BS, AE, PEH, BT, VY. Writing manuscript: FEA, BS, AE, PEH, BT, VY. Critical reviews: FEA, BS, AE, PEH, BT, VY.

Financial disclosure: The authors declared that this study received no financial support.

Editor-in-chief's Note: Five authors of this article, Fatih Erkan Atay, Begüm Söyleyici, Alperen Elibol, Pelinsu Elif Hünkar and Berfin Tan are members of the editorial board of Turkish Medical Student Journal. However, they did not take place in any stage on the editorial decision of the manuscript. The editors who evaluated this manuscript are from other

institutions.

\section{REFERENCES}

1. Mendis S, Armstrong T, Bettcher D et al. Global status report on non-communicable diseases 2014. World Health Organization (WHO):2014.

2. Townsend N, Nichols M, Scarborough P et al. Cardiovascular disease in Europe-epidemiological update 2015. Eur Heart J 2015;36:2696-705.

3. Institute for Health Metrics and Evaluation (IHME). Findings from the Global Burden of Disease Study 2017. Seattle, WA: IHME, 2018. 4. Yeh RW, Sidney S, Chandra $\mathrm{M}$ et al. Population trends in the incidence and outcomes of acute myocardial infarction. N Engl J Med 2010;362:2155-65.

5. Schoen FJ. Morphology, clinicopathologic correlations, and mechanisms in heart valve health and disease. Cardiovasc Eng Technol 2018;9:126-40.

6. Aikawa E, Schoen FJ. Calcified and degenerative heart valve disease. In: cellular and molecular basis of cardiovascular disease. Arterioscler Thromb Vasc Biol 2014;34:2387-93.

7. Schoen FJ, Mitchell RN. The heart. In: Kumar V, Abbas AK, Aster JC, editors. Robbins and Cotran pathologic basis of disease (Ninth edition). Philadelphia PA: Elsevier/Saunders; 2015.p.523-78.

8. Hopkins PN, Williams RR. A survey of 246 suggested coronary risk factors. Atherosclerosis 1981;40:1-52.

9. Dawber TR, Kandel WB, Revotskie N et al. The epidemiology of coronary heart disease - the Framingham enquiry. Proc R Soc Med 1962;55:265-71.

10. Maas AH, Appelman YE. Gender differences in coronary heart disease. Neth Heart J 2010;18:598-602.

11. Yutzey KE, Demer LL, Body SC et al. Calcific aortic valve disease: a consensus summary from the Alliance of Investigators on calcific aortic valve disease. Arterioscler Thromb Vasc Biol 2014;34:2387-93. 12. Stewart BF, Siscovick D, Lind BK et al. Clinical factors associated with calcific aortic valve disease. J Am Coll Cardiol 1997;29:630-4. 13. Kindo M, Hoang Minh T, Perrier S et al. Trends in isolated coronary artery bypass grafting over the last decade. Interactive CardioVascular and Thoracic Surgery, 2016;24:71-6.
14. Perez-Hernandez N, Vargas-Alacron G, Martinez-Rodriguez N et al. The matrix metalloproteinase 2-1575 gene polymorphism is associated with the risk of developing myocardial infarction in Mexican patients. J Atheroscler Thromb 2012;19:718-27.

15. Kapko WS, Krzych $€$. Knowledge on cardiovascular risk factors improves the effectiveness of rehabilitation following acute coronary syndrome. Kardiol Pol 2017;75:344-50.

16. Emral R. Diabetes mellitus and hyperlipidemia. Turkiye Klinikleri J Endocrin 2008;1:38-43.

17. Aronson D, Edelman ER. Coronary artery disease and diabetes mellitus. Cardiol Clin 2014;32:439-55.

18. Law MR, Morris JK, Wald NJ. Environmental tobacco smoke exposure and ischaemic heart disease: an evaluation of the evidence. BMJ 1997;315:973-80.

19. Roerecke M, Rehm J. Alcohol consumption, drinking patterns, and ischemic heart disease: a narrative review of meta-analyses and a systematic review and meta-analysis of the impact of heavy drinking occasions on risk for moderate drinkers. BMC Med 2014;12:182.

20. Wensley F, Gao P, Burgess S et al. Association between c reactive protein and coronary heart disease: mendelian randomization analysis based on individual participant data. BMJ 2011;342:548.

21. Inoue N. Stress and atherosclerotic cardiovascular disease. J Atheroscler Thromb 2014;21:391-401.

22. Rugulies R. Depression as a predictor for coronary heart disease. a review and meta-analysis. Am J Prev Med 2002;23:51-61.

23. Wulsin LR, Single BM. Do depressive symptoms increase the risk for the onset of coronary disease? A systematic quantitative review. Psychosom Med 2003;65:201-10. 